CERN-TH/96-155

hep-ph/9606470

\title{
Charged-Meson Production and Scaling Violations of Fragmentation Functions in Deeply Inelastic Scattering at HERA
}

\author{
Dirk Graudenz[1] \\ Theoretical Physics Division, CERN \\ 1211 Geneva 23, Switzerland
}

\begin{abstract}
We compare recent experimental results for one-particle-inclusive processes in deeply inelastic scattering at HERA with theoretical predictions in nextto-leading-order QCD perturbation theory, and study the factorization scale dependence of cross sections and charged multiplicities. In the future, for the HERA machine running at design luminosity, scaling violations of fragmentation functions permit the measurement of the strong coupling constant. We estimate the size of the statistical error of $\alpha_{s}$ that can be achieved, and study the theoretical error due to the various parton density parametrizations and due to the factorization scale dependence.
\end{abstract}

CERN-TH/96-155

June 1996

*Electronic mail address: Dirk.Graudenz@cern.ch

${ }^{\dagger}$ WWW URL: http://wwwcn.cern.ch/ graudenz/index.htm 


\section{Introduction}

The DESY H1 and ZEUS Collaborations have recently published results for charged-particle production in deeply inelastic electron-proton scattering at HERA [1,2]. In this paper, we calculate, in next-to-leading-order QCD perturbation theory, the $x_{F}$-distribution in the current fragmentation region, employing the recent parametrization of charged-meson fragmentation functions from Refs. [3, 4]. The comparison of theoretical and experimental results looks very encouraging and suggests that a determination of the strong coupling constant $\alpha_{s}$ via scaling violations of fragmentation functions might be feasible. We give an estimate of the statistical error $\Delta \alpha_{s}^{\text {stat }}$ that can be expected for the HERA machine runnning at design luminosity, and study the errors $\Delta \alpha_{s}^{\mathrm{PDF}}$ and $\Delta \alpha_{s}^{\text {scale }}$ due to the uncertainty coming from various parton density parametrizations and choices of the factorization scale, respectively. Except for the dependence on the parton density parametrization, an $\alpha_{s}$-determination via scaling violations has the advantage that, being based on a renormalization group equation, it is model-independent $\mathrm{W}^{\top}$. A drawback is that the size of the effect, being proportional to the logarithm of the energy scale, is small. Compared with $e^{+} e^{-}$annihilation, HERA offers the unique possibility to perform the required measurements at various energy scales at one machine without changing the centre-of-mass energy.

The theoretical basis for the calculation of one-particle-inclusive cross sections is the factorization theorem of perturbative QCD (see, for example, Ref. [5] and references therein). For the process $l P \rightarrow l^{\prime} h+X\left(l, l^{\prime}\right.$ are the incoming and outgoing leptons, $P$ is the incoming proton, $h$ is the observed hadron, and $X$ denotes anything else in the hadronic final stat $\notin^{2}$, the cross section $\sigma$ can be written in the form

$$
\sigma=\int \mathrm{d} \xi f_{i / P}\left(\xi, \mu_{f}^{2}\right) \int \mathrm{d} z D_{h / j}\left(z, \mu_{D}^{2}\right) \sigma_{\text {hard }}^{i j}\left(\xi, z, \mu_{f}^{2}, \mu_{D}^{2}, \mu_{r}^{2}\right)
$$

where $f_{i / P}$ and $D_{h / j}$ denote parton densities and fragmentation functions, $\sigma_{\text {hard }}^{i j}$ is the mass-factorized hard scattering cross section, $\xi$ and $z$ are the momentum fractions of the incident parton $i$ and of the observed hadron originating from the fragmenting parton $j$, and $\mu_{f}, \mu_{D}$ are the corresponding factorization scales. We have also indicated the renormalization scale $\mu_{r}$. The phenomenological distribution functions $f$ and $D$ have to be taken from experiment. The hard scattering cross section $\sigma_{\text {hard }}$ has been calculated in Ref. [6]. For the

\footnotetext{
${ }^{1}$ For example, in the case of $(2+1)$-jet production, the experimental jet rates have to be "corrected" back to the parton level by means of a fragmentation model, thus introducing an additional systematic uncertainty.

2 We identify the four-momenta of particles with their genuine names.
} 
present numerical study, we have used an implementation of a recent recalculation [7]. In leading order, the cross section is given by the process of the naive parton model with a fragmentation function attached to the outgoing quark, see Fig. 11.

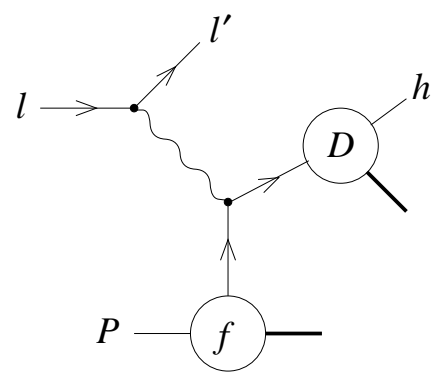

Fig. 1. Feynman diagram corresponding to the leading-order contribution.

In next-to-leading order, virtual corrections have to be added to the $\gamma^{*} q \rightarrow q$ diagram, and the QCD subprocesses $\gamma^{*} q \rightarrow q g$ and $\gamma^{*} g \rightarrow q \bar{q}$ have to be included. After factorization of collinear singularities in the parton cross section and renormalization of the phenomenological distribution functions, the resulting one-particle-inclusive cross section is infrared-finite. A comparison of theoretical and experimental $x_{F}$-distributions is done in the next section. The scales $\mu_{f}, \mu_{D}$ and $\mu_{r}$ are in principle arbitrary. In the leading-order process under consideration, the only physical scale related to the hard scattering process is the photon virtuality $Q=\sqrt{-q^{2}}$, with $q$ the momentum of the exchanged photon. We will therefore identify the factorization and renormalization scales with $Q$, except for the case where we study the scale dependence explicitly.

We will consider only processes with an exchanged virtual photon, and neglect the contributions from an exchanged $Z$ boson. For the comparison with present-day experimental data, this is justified by the restricted range in $Q$. For simplicity, the analysis in Section 3 of the error of a possible measurement of the strong coupling constant via scaling violations of fragmentation functions is done in this approximation as well, even though $Q$ may reach up to $150 \mathrm{GeV}$. We assume this to be sufficient to achieve an estimate of the error, but it is clear that for an experimental analysis at large $Q$ the contributions for an exchanged $Z$ boson have to be included. 


\section{Comparison with Experimental Data}

In this section we compare the longitudinal momentum fraction distribution\$

$$
\rho\left(x_{F}\right)=\frac{1}{\sigma_{\text {tot }}} \frac{\mathrm{d} \sigma}{\mathrm{d} x_{F}}
$$

recently published by the H1 [1] and ZEUS [2] Collaborations with the theoretical next-to-leading-order prediction. Here $\sigma_{\text {tot }}$ is the total cross section for the same cuts on the phase space of the outgoing lepton as for the oneparticle-inclusive cross section $\sigma$. The variable $x_{F}$ is defined to be $2 h_{L} / W$, where $h_{L}$ is the component of the observed hadron's momentum along the direction of the exchanged virtual photon in the hadronic centre-of-mass frame, and $W=\sqrt{(P+q)^{2}}$ is the total hadronic energy. The current direction is defined by the condition $x_{F} \geq 0$.

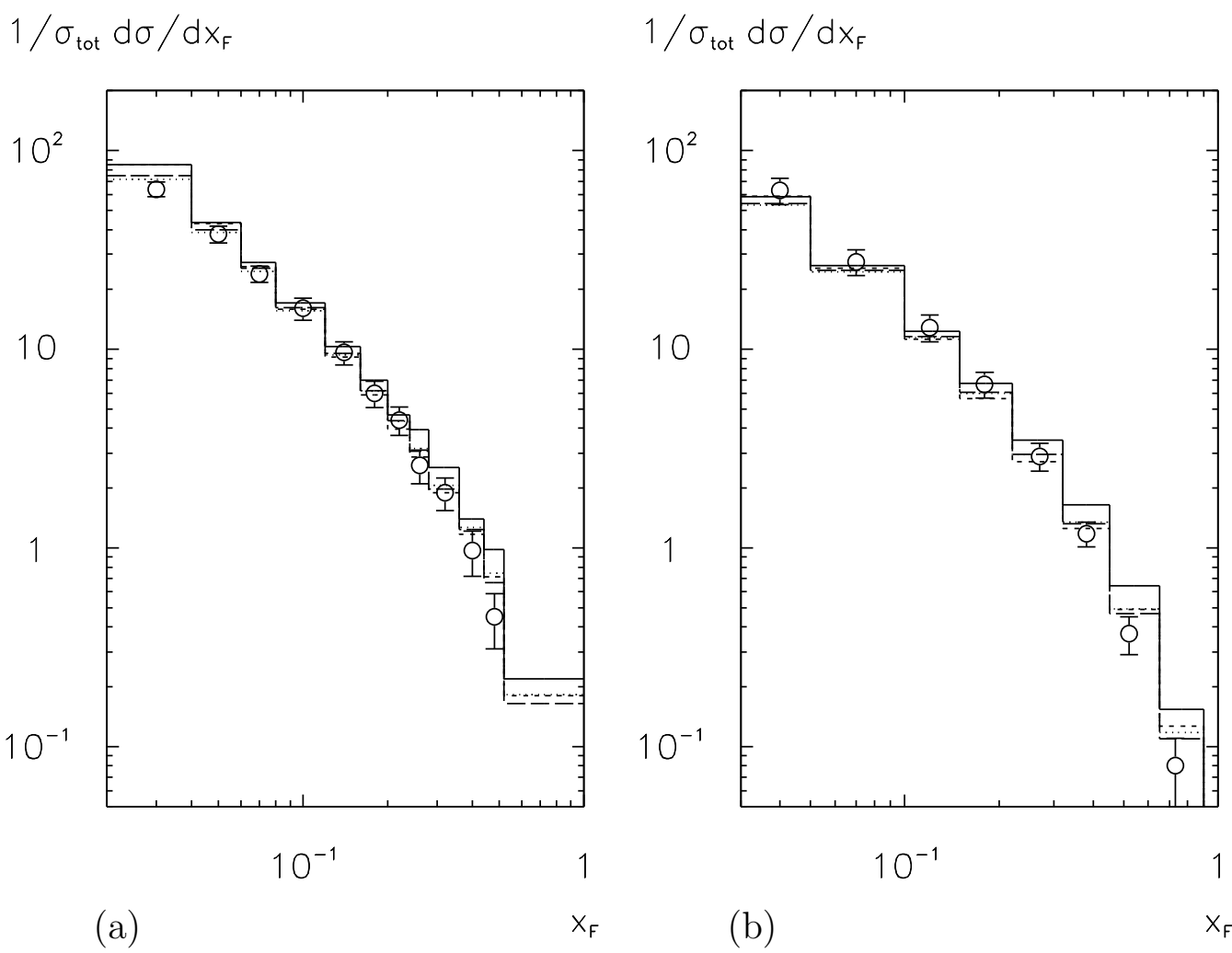

Fig. 2. Distributions in $x_{F}$ in comparison with $H 1$ (a) and ZEUS (b) data. The parton density parametrizations are: GRV LO [-] (with leading-order matrix elements); GRV HO [---], MRS A' [-..], CTEQ 3M [--] (with next-to-leading-order matrix elements).

\footnotetext{
${ }^{3}$ We note that the present calculation does not permit a next-to-leading-order comparison of transverse momentum spectra.
} 
The HERA proton and lepton energies are $E_{P}=820 \mathrm{GeV}$ and $E_{l}=26.7 \mathrm{GeV}$, respectively. The cuts imposed in the experimental analyses are: (a) H1 cuts: $10^{-4} \leq x_{B} \leq 10^{-2}, Q \leq 10 \mathrm{GeV}, 54.77 \mathrm{GeV} \leq W \leq 200 \mathrm{GeV}, E_{l^{\prime}} \geq 14 \mathrm{GeV}$, $157^{\circ} \leq \vartheta_{l^{\prime}} \leq 172.5^{\circ}$; (b) ZEUS cuts: $0.04 \leq y \leq 0.85,3.16 \mathrm{GeV} \leq Q \leq$ $12.65 \mathrm{GeV}, 75 \mathrm{GeV} \leq W \leq 175 \mathrm{GeV}, E_{l^{\prime}} \geq 10 \mathrm{GeV}$. Here $x_{B}=Q^{2} / 2 P q$, $y=P q / P l$, and $E_{l^{\prime}}$ and $\vartheta_{l^{\prime}}$ are the energy and polar angle of the outgoing lepton in the laboratory frame. The experimental data are corrected from the limited detector acceptance for the observed charged particle to the full phase space.

Figure 2 shows the comparison of experimental data with the theoretical prediction for various sets of parton distribution function $\$^{4}$. The employed value for $\Lambda_{\mathrm{QCD}}$ is the one from the parton distribution functions. Charm and bottom quarks are treated as massless flavours in the matrix element, and the flavour threshold in the running coupling constant is assumed to be at the single heavy quark masses. Except for very small and very large values of $x_{F}$, the agreement of theory and experiment is quite satisfactory. The nextto-leading-order corrections are negative for most bins in $x_{F}$, and bring the prediction in better agreement with the data, in particular in the large- $x_{F}$ range. Therefore, the QCD corrections are important to describe the data. For large $x_{F}$, the theoretical prediction is, however, systematically larger than the experimental result, but still within about one standard deviation of the data, depending on the parton density parametrization under consideration. It is expected that the fragmentation function picture breaks down at small $x_{F}$, because soft fragmentation effects, such as particle production between the current and remnant jets, become important. Moreover, the fragmentation function fit in Refs. [3, 4] only takes into account data for $0.1 \leq x_{F} \leq 0.8$. Outside of these bounds, the fragmentation functions are extrapolated. We also wish to note that the experimental data include all produced charged hadrons, whereas the theoretical prediction, due to a lack of a suitable set of fragmentation functions, is restricted to charged pions and kaons.

We finish this section with some remarks concerning the scale dependence of the theoretical prediction. Figure 23 shows the dependence of the cross sections and of the charged multiplicity $\sigma / \sigma_{\text {tot }}$, for the cuts of the ZEUS analysis, as a function of the factorization scales $\mu_{f}$ and $\mu_{D}$, varying individually in the form $\zeta Q$, the other scales being kept fixed at $Q$. A minimum value of $2 \mathrm{GeV}$ for the factorization scales is required. The parton densities are given by the GRV parametrizations. For the one-particle-inclusive cross section, the dependence on $\mu_{f}$ is much flatter in next-to-leading order than in leading order, whereas the dependence on $\mu_{D}$ is slightly larger in next-to-leading order (although it develops an extremum). Including only the scale-compensating

\footnotetext{
4 The parton density parametrizations are: GRV LO and GRV HO [8], MRS A' [9] and CTEQ 3M [10].
} 


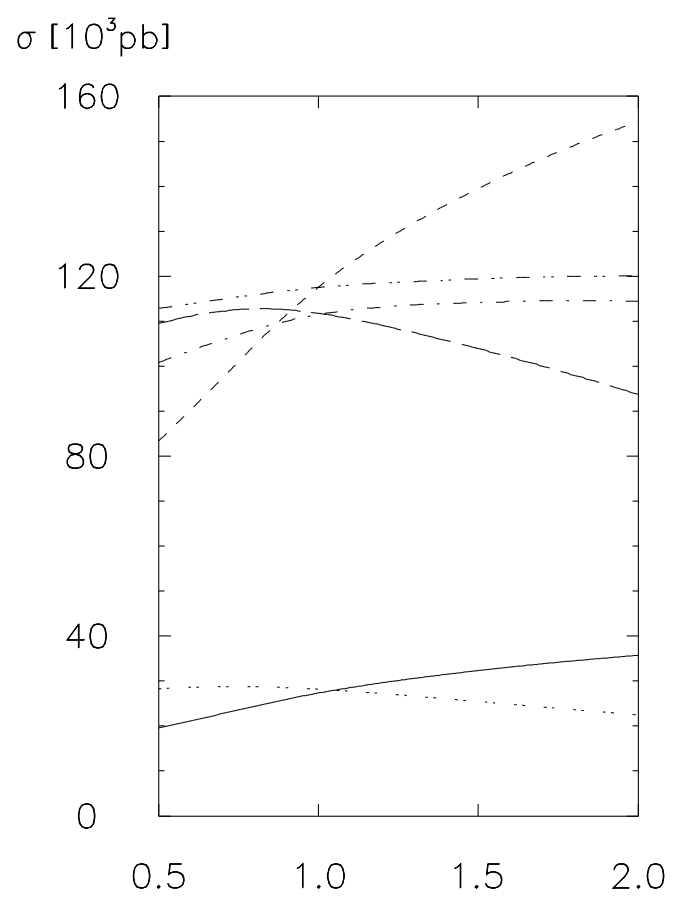

(a) multiplicity

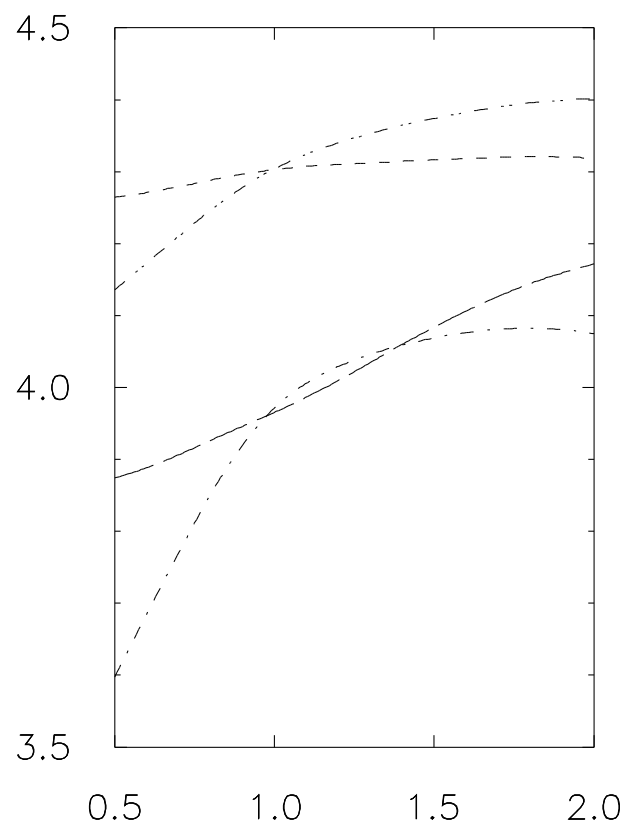

(b)

Fig. 3. Scale dependence of cross sections (a) and multiplicity (b) for the ZEUS cuts. The particular scale set to $\zeta Q$ is given by: (a) $\mu_{f}(-\mathbf{-}), \mu_{D}(-.$.$) in leading order$ and $\mu_{f}(-\mathbf{C}), \mu_{D}(-\cdot-)$ in next-to-leading order for the one-particle-inclusive cross section $\sigma, \mu_{f}$ in leading (-) and next-to-leading $(. . .$.$) order for the total$ cross section $\sigma_{\text {tot }}$; (b) $\mu_{f}(-\mathbf{-}), \mu_{D}(-.$.$) in leading order and \mu_{f}(-\mathbf{-}), \mu_{D}$ (- - - ) in next-to-leading order for the multiplicity. The other scales are fixed to be equal to $Q$.

terms from the next-to-leading-order matrix element proportional to a product of a splitting function and a logarithm in $\mu_{D}^{2}$, the $\mu_{D}$-dependence in next-toleading order is much smaller than the one in leading order. The enhanced $\mu_{D^{-}}$ dependence is therefore a genuine higher-order effect. The $\mu_{f}$-dependence of the total cross section is reduced in next-to-leading order as well. Although the scale dependence of the absolute cross sections looks quite reasonable, the scale dependence of the multiplicity is larger in next-to-leading order than in leading order (Fig. 3b). A similar study shows that the overall scale dependence of the multiplicity is significantly reduced for larger values of $Q$. The impact of the scale uncertainty on an $\alpha_{s}$-measurement will be studied in the next section. The dependence on the renormalization scale, not shown in the figures, is very small, because the running coupling constant enters the matrix elements only in next-to-leading order. 


\section{The Strong Coupling Constant via Scaling Violations?}

The good agreement of the $x_{F}$-distributions of theory and data, as discussed in the previous section, is an encouraging sign for the feasibility of a measurement of the strong coupling constant via scaling violations of fragmentation functions. The scale evolution of fragmentation functions is governed by a renormalization group equation 11 ?

$$
\frac{\partial D_{h / j}\left(z, \mu_{D}^{2}\right)}{\partial \ln \mu_{D}^{2}}=\frac{\alpha_{s}\left(\mu_{D}^{2}\right)}{2 \pi} \int_{z}^{1} \frac{\mathrm{d} u}{u} K_{k \leftarrow j}\left(u, \alpha_{s}\left(\mu_{D}^{2}\right)\right) D_{h / k}\left(\frac{z}{u}, \mu_{D}^{2}\right) .
$$

A multiparameter fit of fragmentation functions for partons into charged hadrons thus permits a determination of the fundamental QCD parameter $\Lambda_{\mathrm{QCD}}$ (or equivalently the strong coupling constant $\alpha_{s}\left(M_{Z}^{2}\right)$ at the mass of the $Z$ boson), if the measurement is performed for several scales $\mu_{D}$. Since the scale evolution depends only on the logarithm of the scale, it is necessary to include a wide range of scales in the fit. The photon virtuality $Q$ is the only scale at hand in the process under consideratior ${ }^{\circ}$, and consequently the analysis will require high statistics, because of the rapidly falling cross section with increasing $Q$.

We study here three potential sources of errors: the statistical error due to a limited number of events, in particular at large $Q$; the error coming from the spread of the theoretical cross sections due to various parametrizations of parton densities, and the uncertainty due to the choice of the factorization scale $\mu_{D}$. The error estimate presented here can, of course, not replace an analysis based on a $\chi^{2}$-fit; it is rather intended to give a first assessment of the sizes of the various uncertainties.

The procedure to obtain an estimate of the statistical error of $\alpha_{s}$ is the following. We fix the fragmentation functions at a scale of $\mu_{0}=2 \mathrm{GeV}$ as the leading-order parametrization of Refs. [3, 国]. We then evolve this input with two different values for $\Lambda_{\mathrm{QCD}}^{(4)}$ of $\{\mathrm{a}\} 0.1 \mathrm{GeV}$ and $\{\mathrm{b}\} 0.2 \mathrm{GeV}$. The corresponding $x_{F}$-distributions $\rho^{\{a\}}$ and $\rho^{\{b\}}$ are determined for these two sets of

5 The evolution kernels $K_{k \leftarrow j}\left(u, \alpha_{s}\right)$ in next-to-leading order can be found in Ref. [12]; see also Ref. [13].

${ }^{6}$ The total hadronic energy $W$ is not directly related to the hard scattering process, because it contains contributions from the proton remnant jet.

7 We have to make an assumption of this kind in order to get an estimate of the dependence of the $x_{F}$-distribution on the value of the strong coupling constant. Since later on we take ratios of the distributions at two different scales (we are only interested in the slope depending on $Q$ ), it is not really important at which scale we identify the distributions. 
fragmentation functions. With the cuts of the ZEUS data analysis unmodified, except for the cut on $Q$, we determine the distributions $\rho^{\{a 1\}}, \rho^{\{a 2\}}, \rho^{\{a 3\}}$, $\rho^{\{b 1\}}, \rho^{\{b 2\}}, \rho^{\{b 3\}}$ for three bins ${ }^{8}$ in $Q:\{1\}[3.16,12.6] \mathrm{GeV},\{2\}[12.6,100] \mathrm{GeV}$ and $\{3\}[100,150] \mathrm{GeV}$. The ratios $\lambda^{\{21\}}=\rho^{\{2\}} / \rho^{\{1\}}$ and $\lambda^{\{32\}}=\rho^{\{3\}} / \rho^{\{2\}}$ for an arbitrary coupling constant $\alpha_{s}$ (taken at the mass of the $Z$ boson) are assumed to depend linearly on $\alpha_{s}$ :

$$
\lambda^{\{i j\}}=\lambda^{\{a i j\}}+\frac{\lambda^{\{b i j\}}-\lambda^{\{a i j\}}}{\alpha_{s}^{\{b\}}-\alpha_{s}^{\{a\}}}\left(\alpha_{s}-\alpha_{s}^{\{a\}}\right) .
$$

For a given luminosity, and under the assumption of a Gaussian error for the event numbers, this allows us to estimate the statistical error $\epsilon_{k}$ of $\alpha_{s}$ for every bin $k$ in $x_{F}$. These individual errors are then combined into a total error $\epsilon$ according to $\epsilon=1 / \sqrt{\sum_{k}\left(1 / \epsilon_{k}^{2}\right)}$. To obtain explicit numerical values, we use the CTEQ 3L parametrization [10] for the parton densities (for simplicity, we work in leading order). The integrated luminosity is assumed to be $250 \mathrm{pb}^{-1}$ (the HERA design luminosity, per experiment, integrated over five years). For the analysis based on bins $\{1\}$ and $\{2\}$, we obtain a statistical error of $\alpha_{s}\left(M_{Z}^{2}\right)$ of \pm 0.0007 , and for the bins $\{2\}$ and $\{3\}$, the statistical error is \pm 0.027 .

Another problem of the extraction of $\alpha_{s}$ is the dependence of the theoretical cross sections on the parton density parametrization. Taking the ratio of oneparticle-inclusive and total cross sections leads to a substantial cancellation, but a certain residual dependence remains. To estimate the size of this effect, we determine the spread of the results for $\alpha_{s}\left(M_{Z}^{2}\right)$ depending on the next-toleading-order parton densities from Refs. 8, 9, 10]. For the bins $\{1\}$ and $\{2\}$, the spread is \pm 0.017 , and for the bins $\{2\}$ and $\{3\}$, the spread is \pm 0.005 . Future global fits of parton densities including improved HERA data should reduce this systematic uncertainty.

Finally, we discuss the dependence of $\alpha_{s}$ on the choice of the factorization scale $\mu_{D}$. To obtain an estimate, the ratios $\lambda$ are determined for the three choices $Q / 2, Q$ and $2 Q$ of this scale. The change of cross section has for consequence a variation in the extracted $\alpha_{s}\left(M_{Z}^{2}\right)$-value of \pm 0.013 and \pm 0.011 for the combinations of the bins $\{1\},\{2\}$ and $\{2\},\{3\}$, respectively.

\footnotetext{
${ }^{8}$ It turns out that the dependence on the parton densities is unacceptably large for smaller values of $Q$ due to the large spread of parametrizations at small $x$. Bin $\{1\}$ is the $Q$-range of the original ZEUS analysis. The other two bins are chosen such that one of them includes data only at very large $Q$. We expect that bin optimization will lead to a smaller overall error by balancing statistical and systematic errors; this is, however, beyond the scope of the present paper.
} 


\section{Summary and Conclusions}

We have calculated, in next-to-leading order, the $x_{F}$-distribution for the production of charged mesons in deeply inelastic scattering at HERA. The comparison with experimental data is satisfactory. The dependence of the theoretical cross section prediction on the factorization scale $\mu_{f}$ is considerably reduced, whereas the scale dependence of the charged multiplicity is increased. The $\mu_{D}$-dependence of the next-to-leading-order cross sections, although slightly larger than in leading order, is small. The cross section results can therefore be considered to be reliable for a quantitative test of QCD.

As a particular example, we have estimated the statistical and systematic errors (due to theoretical uncertainties) for an extraction of the strong coupling constant via scaling violations of fragmentation functions. We find, for the HERA machine running at design luminosity for five years, $\Delta \alpha_{s}^{\text {stat }} \approx$ $\pm 0.0007 / \pm 0.027, \Delta \alpha_{s}^{\mathrm{PDF}} \approx \pm 0.017 / \pm 0.005$ and $\Delta \alpha_{s}^{\text {scale }} \approx \pm 0.013 / \pm 0.011$ for two specific sets of cuts. It is expected that there is still some room for improvement by means of bin optimization. We have not considered experimental systematic uncertainties. They will also contribute to the error on $\alpha_{s}$. The dominant systematic error in the experimental $x_{F}$-distributions comes from the boost of the particle momenta from the laboratory system into the hadronic centre-of-mass system. For a measurement of $\alpha_{s}$ it may therefore be advantageous to define a suitable observable in the laboratory system, to circumvent this source of systematic uncertainty.

Compared with the present error $\Delta \alpha_{s}=0.006$ of the world average, the errors estimated here are large. It should be kept in mind, however, that a reduction of $\Delta \alpha_{s}^{\mathrm{PDF}}$ can be expected because of improved fits of parton densities, in particular in the small- $x$ region. It might also be possible to reduce the dependence on the parton density by performing the analysis in the Breit frame [14]. A reduction of $\Delta \alpha_{s}^{\text {scale }}$ would require a higher-order calculation or a physical scheme for the choice of the factorization scales. A measurement of $\alpha_{s}$ at HERA via scaling violations of fragmentation functions is worth doing because it is an independent quantitative test of QCD and, more important, because it complements other methods for an $\alpha_{s}$-determination in deeply inelastic scattering, such as the measurement via $(2+1)$-jet rates and via scaling violations of structure functions.

\section{Acknowledgements}

I wish to thank N. Brook, M. Kuhlen and N. Pavel for discussions and for comments on the manuscript, Ch. Berger and T. Doyle for discussions, and J. Bin- 
newies for clarifying remarks concerning the parametrizations of Refs. [3, 4 . This work was supported in part by a Habilitandenstipendium of the Deutsche Forschungsgemeinschaft.

\section{References}

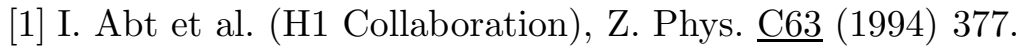

[2] M. Derrick et al. (ZEUS Collaboration), Z. Phys. C70 (1996) 1.

[3] J. Binnewies, B.A. Kniehl and G. Kramer, Z. Phys. C65 (1995) 471.

[4] J. Binnewies, B.A. Kniehl and G. Kramer, Phys. Rev. D52 (1995) 4947.

[5] J.C. Collins, D.E. Soper and G. Sterman, in: Perturbative Quantum Chromodynamics, ed. A.H. Mueller, World Scientific (Singapore, 1989).

[6] G. Altarelli, R.K. Ellis, G. Martinelli and S.Y. Pi, Nucl. Phys. B160 (1979) 301.

[7] D. Graudenz, Heavy-Quark Production in the Target Fragmentation Region, CERN-TH/96-52, in preparation.

[8] M. Glück, E. Reya and A. Vogt, Z. Phys. C67 (1995) 433.

[9] A. Martin, R. Roberts and J. Stirling, Phys. Rev. D51 (1995) 4756.

[10] CTEQ Collaboration, Phys. Rev. D51 (1995) 4763.

[11] R. Baier and K. Fey, Z. Phys. C2 (1979) 339.

[12] G. Curci, W. Furmanski and R. Petronzio, Nucl. Phys. B175 (1980) 27.

[13] P. Nason and B.R. Webber, Nucl. Phys. B421 (1994) 473.

[14] N. Brook and T. Doyle, private communication. 